\title{
Acercamiento onomástico al nombre de las bandas de Heavy Metal
}

\section{Onomastic approach to the name of Heavy Metal bands}

\author{
Miguel Reyes Contreras \\ Universidad de Ixtlahuaca CUI \\ https://orcid.org/0000-0003-3816-9189 \\ reyescontramiguel@gmail.com
}

\section{Resumen}

El acto de nombrar es considerado en cierto modo un ritual. Parte de una lista de opciones y termina en una etapa en la que se narra el proceso. Cualquier ente que tiene un nombre, lo recibe para ser individualizado y ser considerado único por lo menos en el espacio circundante y no es la excepción en el proceso de nombrar una banda musical, concepto conocido como crematónimo. El propósito de este texto es presentar un análisis onomástico, sobre un corpus de nombres de bandas de varios subgéneros de heavy metal y conocer la estructura lingüística de los nombres, las lenguas de nominación y aspectos onomásticos asociados con influencias sociales, religiosas, culturales, literarias, etc. y la clasificación y discusión en la nomenclatura onomástica. Bajo un enfoque cuantitativo y desde los puntos de vista lingüístico y onomástico se analiza un corpus de nombres de 844 bandas de Heavy Metal (de todo el mundo), un género que ha sabido construir su propia subcultura con reglas, modas, discurso propio y sus rituales de nominación. El análisis revela influencias culturales, literarias, históricas, religiosas y lingüísticas en la nominación de las bandas y una amplia variedad de construcciones morfológicas y sintácticas en los nombres. Encontramos que esta variedad de referencias vertidas en un nombre de una agrupación es un entramado de conexiones sociales, lingüísticas y culturales y no deben ser estudiados solo desde la perspectiva musicológica o antropológica, sino también desde los ámbitos lingüísticos, sociales y culturales.

Palabras clave: Crematónimo, Heavy metal, Onomástica, intercategorías.

\begin{abstract}
The act of naming is considered a certain ritual. It is rooted in a list of options and ends in a stage where the process is narrated. Any object which bears a name, bears it to be seen as individual and unique, at least in the surroundings, and the process of naming a music band is no exception. The concept used for this process is Chrematonymy. The main aim of this text is to present an onomastic overview, out of a several subgenres of heavy metal band name corpus in order to explore the linguistic structure of such names, languages chosen for naming and onomastic aspects associated to social, religious, cultural, literary influences and also to classify and discuss based onomastic nomenclature. Based on a quantitative approach and from the linguistic and onomastics point of view, a corpus 844 Heavy Metal bands' names from all over the world, a genre which constructed its own subculture with their own rules, fashion, discourse and naming rituals. Our analysis reveals cultural, literary, historical, religious and linguistic in band naming as well as an ample variety of morphological and syntactic constructions in the catalog of names. We can find that the variety of references in the name of a band is a network of social, linguistic and cultural connections and should not be only studied from the musicology or anthropology' perspective, but also as a linguistic, social and cultural phenomenon.
\end{abstract}

Key words: Chrematonym, Heavy metal, Onomastics, intercategories. 
El Heavy Metal (HM en lo que sigue), por lo general se ha considerado un género marginal pues se le asocia con asuntos oscuros, obscenos y violentos. Los que estudian y los aficionados a estos géneros tienen opinión diferente y ven en el tema un vastísimo campo de estudio que puede abordarse desde la interdisciplina. Este trabajo presenta un breve análisis sobre algunos procesos de nominación de bandas, comenzamos con algunos datos sobre el acto de nombrar y una discusión sobre el crematónimo. A continuación, presentamos la metodología y los resultados. El objetivo general es presentar un análisis onomástico, sobre un corpus de nombres de bandas de varios subgéneros de HM (de todo el mundo) y conocer la estructura lingüística de los nombres, las lenguas de nominación y aspectos onomásticos asociados con influencias sociales, religiosas, culturales, literarias, etc. y la clasificación y discusión en la nomenclatura onomástica.

La existencia de encuentros de estudios sobre Heavy Metal, del Seminario Permanente de Estudios sobre Heavy Metal (Instituto de Investigaciones Antropológicas, UNAM) o del International Seminar of Heavy Metal Music Studies prueba la notable presencia de este género musical en la academia a nivel nacional e internacional. También representa una oportunidad de llevar a la onomástica a otros espacios de investigación y de trabajo multi e interdisciplinar.

\section{Algunos apuntes sobre el heavy metal}

Phillips y cogan (2016: 3) afirman que el género nace en los años sesenta, aunque depende de qué banda se tome como punto de referencia. También expresan que el HM es tanto un subgénero del rock and roll y al mismo tiempo "una manifestación de la subcultura con reglas, rituales, formas de pensar, incluso, modas". En su Encyclopedia of Heavy Metal Music, los autores trazan de manera breve los orígenes de este subgénero tanto del término "heavy metal" como de sus raíces histórico-musicales. Con respecto a lo último, afirman que el blues y la música clásica son las mayores influencias del HM. Del blues, los artistas británicos en particular se inspiran en músicos de blues como Muddy Waters y Howling Wolf, quienes son imitados por Eric Clapton y Jeff Beck en cuanto a la distorsión de guitarras para decantar en los sonidos característicos de bandas como Black Sabbath o Led Zeppelin. Por el lado de la influencia de la música clásica, músicos como Rick Wakeman y Keith Emerson, con formación de conservatorio, 
llevaron estos conocimientos al rock progresivo y a estructuras compositivas más complejas (PHILLIPS \& COGAN, 2016: 3). Hay ejemplos como el de Ritchie Blackmore (Deep Purple), Uli Jon Roth (Scorpions) e Yngwie Malmsteem que fusionaron elementos complejos de la música clásica, incluso estos dos últimos tienen reinterpretaciones en HM de piezas de Vivaldi, Niccolo Paganini, Beethoven y Bach.

Hadžajlić (2018: 129) dice que el HM es "A specific, alternative music genre that exists on the fringe of popular music, where it is classified by its own culture: musical style, fashion, philosophy, symbolic language and political activism ${ }^{1}$ ". Retoma también del Oxford Dictionary of New Words que el HM es

a style of loud energetic rock music, characterized by the use of heavily amplified instruments (mostly guitars, bass guitars and drums), intense and fast beats (which refers to active, intense, fast rhythm and pulse), often energetic and spectacular performance and a generally rough and aggressive music style; as a later stage of the development of hard rock (Hadžajlić, 2018: 129)².

Aunado a lo anterior, en el desarrollo mismo del género, este autor menciona varios de subgéneros en los que ha desembocado, entre los que destaca el protometal, el NWOBHM ${ }^{3}$, speed metal, trash metal, black metal, gothic metal, death metal, power metal, jazz metal, folk metal, party or pop metal, glam metal, metal-core, gore metal, punk metal, doom metal, progressive metal, industrial metal, grind-core, nu metal, postmetal. A lo anterior, Angeler (2016: 2) define al HM como:

A fast-evolving form of the auditory arts belonging to the genre of rock music. Metal has roots in blues rock and psychedelic rock and originated in the late 1960s and early 1970s, mainly in the United Kingdom and the USA. Heavy metal has diversified into more than 20 subgenres with characteristic vocal, rhythm and instrumentation. ${ }^{4}$

Estos subgéneros se clasifican de acuerdo con tres elementos característicos: la voz, el ritmo y la instrumentación, pues "[n]owadays it spans a wide range of song architectures, lyric themes, and instrumentation, including distorted guitars, emphatic

\footnotetext{
${ }^{1}$ en específico, un género alternativo de música que existe en la periferia de la música popular, donde se le clasifica por su propia cultura: estilo musical, moda, filosofía, lenguaje simbólico y activismo político (traducción propia) ${ }^{2}$ Un estilos de música rock de volumen alto y enérgico, que se caracteriza por usar instrumentos altamente amplificados (en su mayoría son guitarras, bajo y batería), beats rápidos e intensos (lo que se refiere al ritmo rápido, intenso y activo, así como al pulso), con frecuencia asociado a presentaciones enérgicas y espectaculares y un estilo musical generalmente áspero y agresivo; es una etapa tardía en el desarrollo del hard rock (Traducción propia).

${ }^{3}$ New Wave Of British Heavy Metal (La nueva ola de Heavy Meta Británico).

${ }^{4}$ Una forma de rápida evolución de las artes auditivas que pertenece al género de la música rock. Le Metal tiene raíces en el blues y el rock psicodélico y se origina a finales de los 60 y principios de los 70, principalmente en el Reino Unido y en los Estados Unidos. El Heavy Metal se ha diversificado en más de 20 subgéneros con características vocales, rítmicas e instrumentación. (Traducción propia)
} 
rhythms, dense bass-and-drum sound, and vigorous vocals..", 5 por lo que se ha diversificado a partir del "metal clasico" en alternative metal, avant-garde metal (avantmetal, experimental metal), black metal, Cello metal, Crust punk, Death metal, Doom metal, Folk metal (Celtic, Pirate, Medieval metal), glam metal (hair metal, sleaze metal, pop metal), Gothic metal, Grind core, Industrial metal, latin metal, metal core, neoclassical metal (Shred metal), New German hardness, Nintendo core, post metal, power metal, progressive metal, speed metal, stoner metal (Stoner rock, desert rock), symphonic metal, trash metal, classic (traditional) heavy metal y algunos otros géneros asociados como Christian metal, dark metal, extreme metal y pagan metal (ANGELER, 2016: 5-10). Los diversos subgéneros, los temas, los seguidores, investigadores de varias disciplinas, etc, constituyen lo que también Angeler (2016: 4) denomina sistema complejo pues se les puede estudiar desde la musicología, sociología, antropología, psicología social, etc. La lingüística parece quedar fuera de este entramado; sin embargo, el nombre de una banda es parte del discurso (tanto mediático, como el poético, es decir, la creación de la lírica de cada subgénero) y este es el que mantiene la conexión entre los diversos componentes del sistema.

En el HM, "fashion and style, however, just like the music, are constantly transforming and evolving into distinct subgenres and subcultures with their own evolving rules and rituals" (PHILLIPS \& COGAN, 2016: 3). Esta idea está evidentemente asociada con la moda de nombrar una banda. En los subgéneros del HM, los nombres implican oscuridad, aspectos sombríos o cualidades negativas: para expresarlas se usan unidades léxicas simples (Immortal, Kataklysm, Underoath, Entombed, Maelström), frases nominales (Mad Season, At The Gates, Morbid Angel) o incluso oraciones (Devil Wears Prada, Between The Buried And Me, The Sins of thy Beloved...) Estos ejemplos también mantienen la cohesión discursiva del género.

\section{Sobre el acto de nombrar}

Nombrar no es un acto fortuito e independiente, sino que obedece a varias dinámicas sociales; Kallio (2016: 144) afirma que “nombrar es Inspiración, Comparación, Elección y Narración”, lo que en palabras de Aldrin (2011: 35) es un entramado de eventos que

\footnotetext{
${ }^{5}$ en la actualidad se expande en un espectro amplio de arquitecturas de la canción, de temas líricos e instrumentación, incluyendo guitarras distorsionadas, ritmos enfáticos, sonido denso del bajo y la batería y vocalizaciones vigorosas. (Traducción propia)

${ }^{6}$ La moda y el estilo están en constante transformación y evolucionando en distintos subgéneros y subculturas desarrollando sus propias reglas y rituales" (Traducción propia).
} 
van desde el momento de la elección a la fase narrativa sobre la elección del nombre (fig. 1). En el proceso de nombrar primero debe haber una lista de opciones que tiene fuentes e influencias de los medios de comunicación, de la línea familiar, de la creatividad misma, etc. Luego se deben sopesar pros y contras de esos nombres y finalmente elegir. Es una decisión difícil pues el nombre que se elige será la primera carta de presentación y se portará por toda la vida. Esta dificultad de elegir y las vicisitudes pasadas para hacerlo es lo que se cuenta en la fase narrativa.

Figura 1. El proceso de nominación (traducido de ALDRIN, 2011: 35)

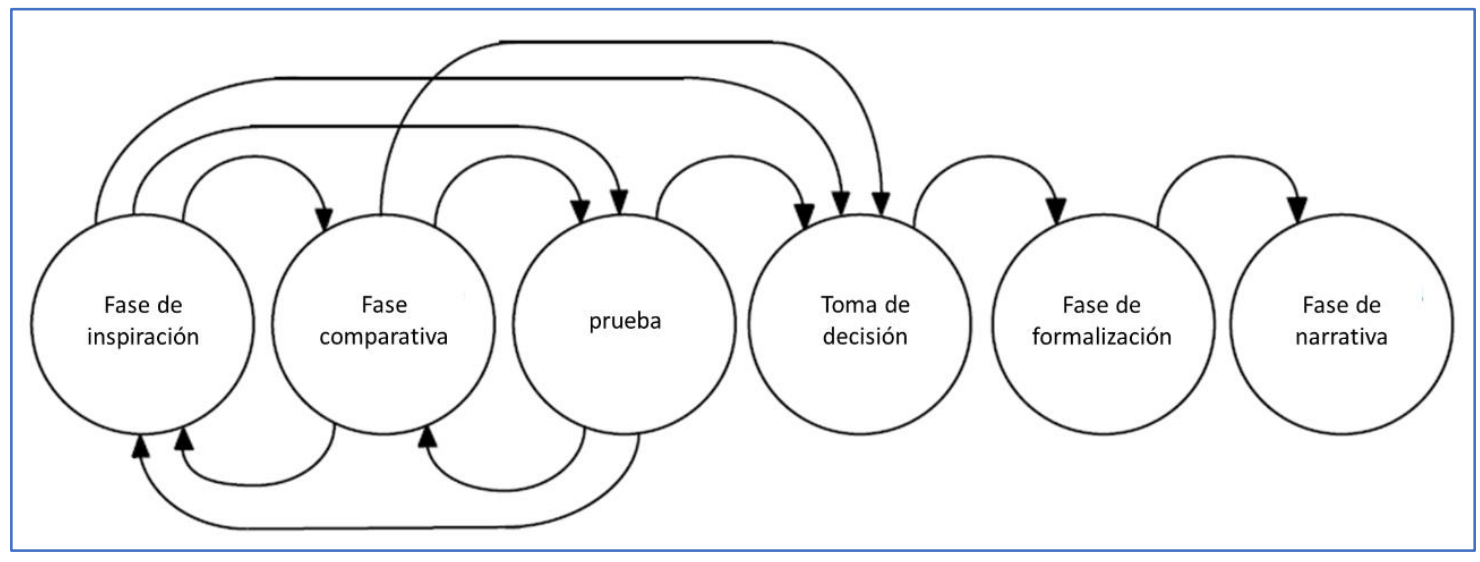

Este proceso aplica, por lo general en el llamado "proyecto parental” (OFFROY: 1982 apud JIMÉNEZ. 2020:176), en el que, haciendo la analogía, los encargados de nombrar a una banda "eligen el nombre de acuerdo con la idea que se han construido de estos incluso antes de que nazca el proyecto, es decir [...] con el imaginario que [...] se han creado [del producto] a partir de la introyección que ellos mismos tienen de las normas sociales y culturales de su época y comunidad" (JIMÉNEZ, 2020: 176). En relación con el tema de este texto, el nombrar a una banda, los nombres, que pueden ser de diversa índole (sustantivos, adjetivos, frases nominales, oraciones, etc.), se convierten en nombres propios que encajan en la categoría de crematónimos y, por lo tanto, sufren un proceso de por el que se mueven "entre la función propia y común de los nombres en otros términos, lo que podría ser el paso “intercategorial"” (LÓPEZ FRANCO, 2020: 212). López Franco analiza cómo una variedad de elementos léxicos (nombres de plantas, adjetivos, sustantivos) que no son estrictamente nombres propios se convierten en nombres de persona, por lo que se observa un cambio de categoría. En la nominación de una agrupación musical, a diferencia del trabajo de López Franco, el proceso es a la inversa puesto que un nombre propio ya establecido en el onomasticón de una lengua se convierte en el referente para un producto comercial. Cuando un grupo de músicos 
deciden formar una agrupación musical, deberán también pasar por las cuatro fases que propone Aldrin ${ }^{7}$ y además agregar el componente simbólico pues la carga semántica del nombre (como se ha mencionado, son connotaciones negativas, por lo general) pues este debe ser acorde con la ideología, las reglas y el discurso del HM. En este sentido, "[E]n el valor simbólico del nombre [...] se pueden identificar igualmente algunos elementos que tienen relación con la identidad y el patrimonio familiar, lingüístico y cultural. La presencia de estos elementos favorece también la circulación entre subcategorías" (LÓPEZ FRANCO, 2020: 218).

\section{Sobre el crematónimo}

Tal vez nombrar un banda no sea tan difícil como poner el nombre a una persona o a una mascota, o quizá sea más difícil porque al final de cuentas, el nombre de la banda, independientemente del éxito alcanzado, terminará convirtiéndose en una marca, es decir, en capital, y actuarán como recurso en la producción de bienes y servicios y como activos en sí mismos, sobre todo en nombres que se han vuelto un nombre comercial (BOERRIGTER \& NIJBOER, 2012: 3). Por lo general, hay una distinción entre nombre comercial y marca (sobre todo porque este último va, en muchos casos, asociado a un logotipo) (RENGIFO, 2013: 187). Además, el nombre de marca es una interrelación entre la lingüística y el marketing (factores linguiísticos y corporativos) (GONZÁLEZ DEL RÍO, AMPUERO, JORDÁ y MAGAL, 2011).

El nombre de una banda pertenece a la categoría de crematónimo, es decir, "el nombre comercial o el nombre con el cual se identifica en el comercio a un producto o la empresa que lo produce" 8 (trad.mía) (CAFFARELLI, 2011). En el diccionario terminológico del International Council of Onomastic Sciences (ICOS) se define como el "Nombre de un objeto o institución político-económico o comercial o cultural" (2020). Se podría decir que se trata de una categoría para todo lo que no encaje en los nombres de personas o de lugares, lo cual vuelve muy complejo el término pues hay demasiados objetos e instituciones que tienen un nombre. La palabra crematónimo deriva del griego

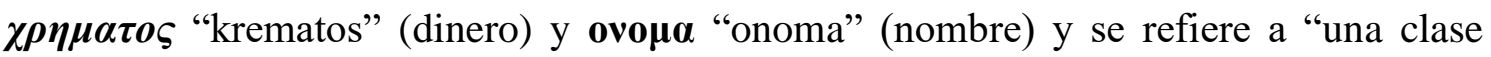

\footnotetext{
${ }^{7}$ Algunos de los nombres de las bandas y sus significados pudieron conseguirse en la información que proporcionan las mismas bandas a la página metal archives (https://www.metal-archives.com), que alberga sus datos. Esto puede considerarse la fase narrativa.

8 "Si dice nome commerciale o marchionimo il nome con cui è noto in commercio un determinato prodotto o il nome dell'azienda che lo produce."
} 
especialmente compleja de nombre propio (que hace referencia tanto a productos tangibles como intangibles de la actividad humana) y que se encuentra al margen de la disciplina de la onomástica (TORT I DONADA y MONTAGUT I MONTAGUT, 2014: 93-94). Gálová (2011: 212) afirma que los crematónimos pertenecen a los nombres literarios propios de un grupo denotativo que incluye los antropónimos, los zoónimos, fitónimos, topónimos o crematónimos y que poseen características como animadoinanimado, auténticos o ficticios, hablado- no hablado. Es escurridizo el concepto; por ejemplo, cuando se trata del nombre de un establecimiento comercial el nombre puede ser una marca, un topónimo o un objeto (REYES CONTRERAS, 2019: 64), en el caso de una banda, una marca asociada a una imagen gráfica (logo).

De este modo el crematónimo es el aspecto onomástico más complejo puesto que abarca diversos espectros de la realidad. Nombramos fraccionamientos, organizaciones públicas, libros, programas de televisión, video juegos, centros comerciales, obras de arte (El Guernica, El David, La Pietá), incluso nombres de lugares y de persona como marca (un Picasso, un Tiziano). También damos nombres a eventos (como Lollapalooza, Vive Latino, Knotfest) y la lista se extiende si de individualizar se trata. Sin embargo, como nos ocupa en este texto, nos centraremos en los procesos de nombrar a bandas de música.

Figura 2. Las dimensiones de la onomástica (elaboración propia)

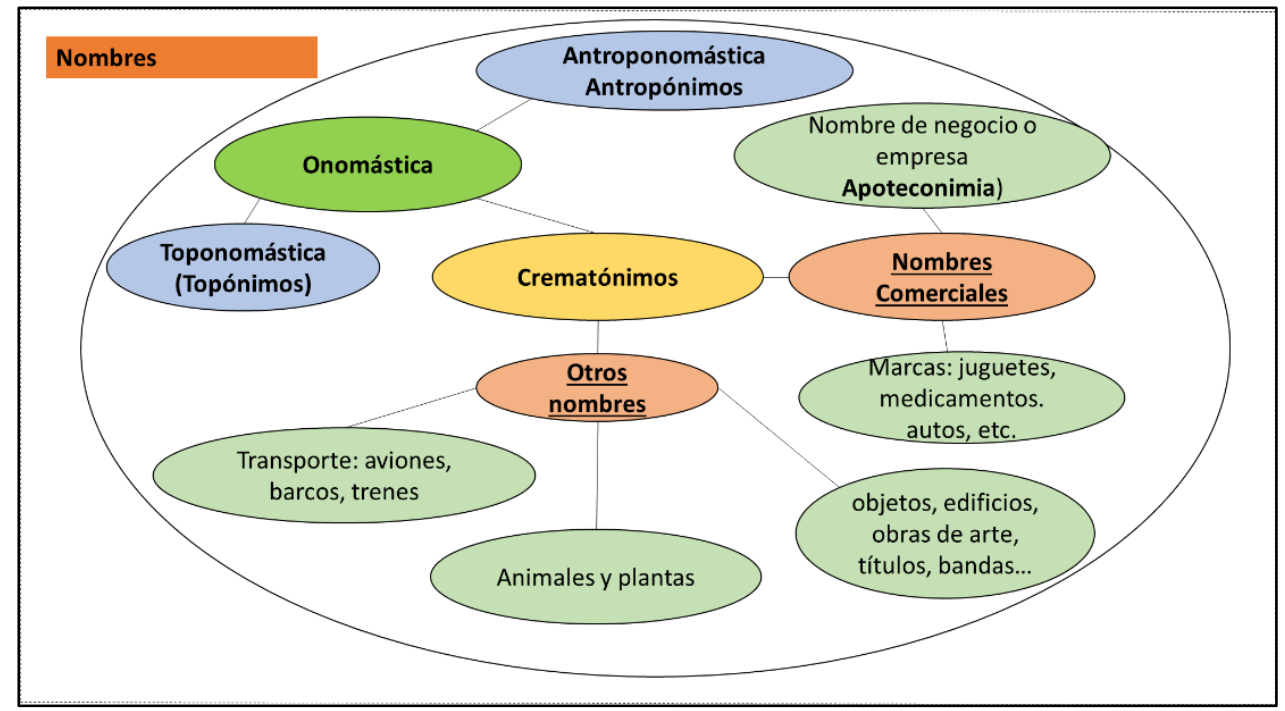

\section{Nombrar a una banda}

Cuando se analizan los nombres de las bandas, podemos conocer tendencias en relación con la estructura lingüística de los nombres, las lenguas de nominación y aspectos sociolingüísticos con relación a las influencias sociales, religiosas, culturales, 
etc. Los nombres de las bandas se relacionan con el género o casi es posible clasificarlos a partir del nombre. En los años 60 y 70, principalmente, los nombres de las bandas hacían referencia morfológica a la agrupación con el artículo y la marca de plural: The Crickets, The Yardbirds, The Beatles, The Blue Notes, los Dandys, Los Locos del Ritmo. Algunas tienen nombres más creativos como Humble Pie, The Who, Mott the Hoople, Procul Harum, Pearl Jam. Otras tienen inspiración histórica o literaria como The Ivy League ${ }^{9}$, Amen Corner, Jethro Tull, H. P. Lovecraft, Isildur's Bane, Marilyn Manson.

Hay gran variedad. La música popular mexicana tiene nombres de agrupaciones en distintos géneros como Los Yonics, Los Caminantes, La Dinastía de Tuzantla, Los Players, La Changa, Los Tigres del Norte, etc. Hay nombres creativos en el rock mexicano como Caifanes, Zoé, Café Tacuba, La Barranca. En el progresivo pueden notarse también influencias literarias y los nombres tienen un aura de misticismo: Cerebrum, Cerebus Effect, Cervello, Chac Mool, Chain, Chameleon, Chaneton, Changes, Chaos Code, Chase, Chase The Sunset ${ }^{10}$. En el HM también hay variedad de influencias. Expondremos esto en las siguientes secciones.

\section{La metodología}

El propósito de este texto es presentar un análisis onomástico, sobre un corpus de nombres de bandas de varios subgéneros de HM y conocer la estructura lingüística de los nombres, las lenguas de nominación y aspectos onomásticos asociados con influencias sociales, religiosas, culturales, literarias, etc. y la clasificación y discusión en la nomenclatura onomástica.

Para el corpus se tomaron los datos de la página darklyrics.com (consultado en octubre de 2019), un sitio web que alberga letras de HM de más de 13800 álbumes de más de 4500 bandas ${ }^{11}$ de todo el mundo. La página alberga los nombres de las bandas en orden alfabético y las letras de los álbumes se presentan en orden cronológico de lanzamiento al mercado. Se decidió tomar todos los nombres de bandas con la letra A, al ser la más numerosa y, por lo tanto, representativa del corpus total, pues las 844 muestras representan el $18.75 \%$ de las bandas en la página. Estos nombres se clasificaron con una

\footnotetext{
${ }^{9}$ La Ivy League (Liga Ivy o Liga de la Hiedra) es una conferencia deportiva de la NCAA de ocho universidades privadas del noreste de los Estados Unidos. El término Ivy League se usa típicamente más allá del contexto deportivo para referirse a las ocho escuelas como un grupo de universidades de élite con connotaciones de excelencia académica, selectividad en las admisiones y elitismo social (https://es.wikipedia.org/wiki/Ivy League)

${ }^{10}$ www.progarchives.com

11 http://www.darklyrics.com/
} 
base de datos de Excel para el análisis. Se elaboraron columnas que contienen el nombre de la banda, su estructura, el número de componentes léxicos, su estructura sintáctica, la lengua de cada componente y la clasificación onomástica (así como el origen de la referencias onomástica y notas).

Los datos sobre el significado, la veracidad del nombre y desambiguación en cuanto a nacionalidad, uso y significados se comprobaron en The Metal Archives. Encyclopaedia Metallum, un sitio que proporciona información de 139322 bandas a nivel mundial. Los datos que alberga son el país de origen, su ubicación, el estatus, año de formación, el género, los temas de su lírica, su disquera, el periodo de actividad (en caso de estar inactiva) y datos adicionales como su historia y en ocasiones, notas sobre el nombre de la banda ${ }^{12}$. Varios de los datos sobre los nombres se comprobaron en bases de datos y en información proveniente de la web, principalmente Wikipedia, enciclopedias especializadas, diccionarios (Oxford y Merriam Webster's), así como bases de datos sobre nombres de personas como Behind the name ${ }^{13}$.

\section{Algunos resultados}

En primer lugar, la columna "origen" se centra en el país de procedencia de la banda y revela la gran prolijidad del ambiente musical en los Estados Unidos. Como se ha mencionado en la sección anterior, los datos de las bandas se corroboraron en bases de datos como Encyclopaedia Metallum y en páginas oficiales o sitios promocionales de las bandas (Facebook o Spotify) De los 10 países que muestran más actividad musical en el heavy metal, claramente observamos que los Estados Unidos lideran la escena, seguidos de Alemania, Suecia y Finlandia. En contraparte, en el corpus encontramos a Andorra, Bolivia, Egipto, Eslovaquia, Eslovenia, Islandia, Jordania, Luxemburgo, Macedonia, Malta, Marruecos, Nepal, República Dominicana, Rumania y Venezuela con un solo representante en la escena (figura 3).

Fig. 3. La distribución por países (elaboración propia)

\footnotetext{
12 https://www.metal-archives.com/

13 www.behindthename.com
} 
Onomástica Desde América Latina, n.3, v.2, janeiro - junho, 2021, p. 59 - 81 ISSN 2675-2719 https://doi.org/10.48075/odal.v0i0.26062

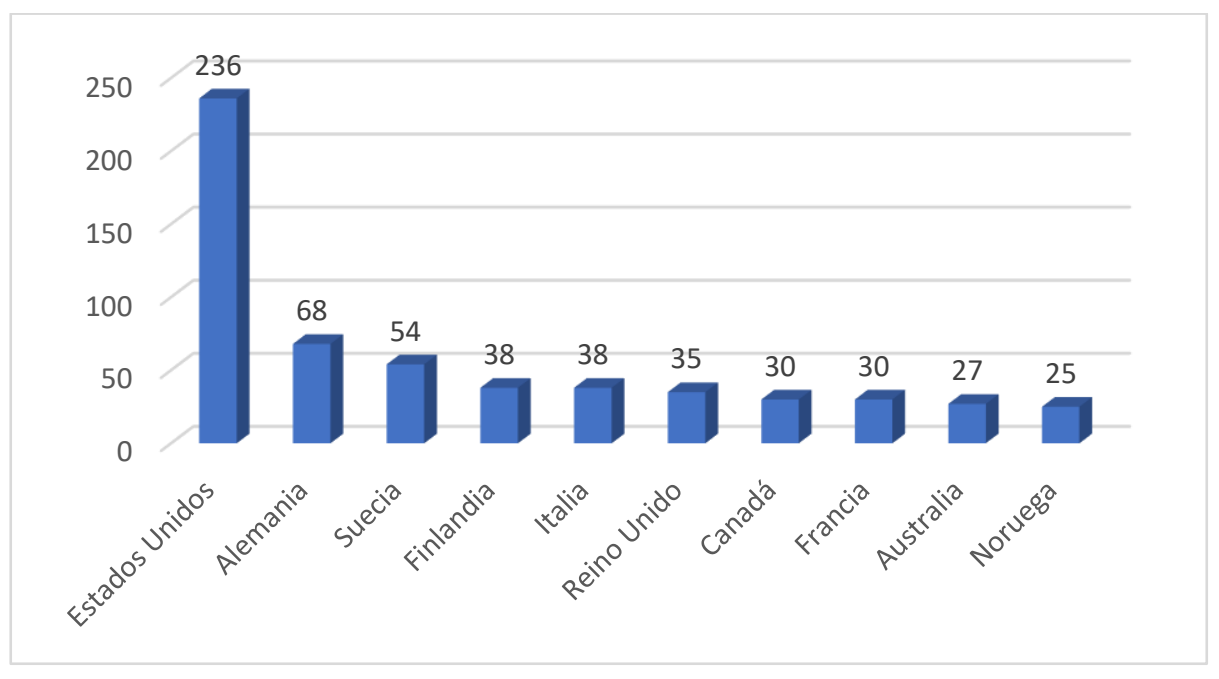

Estados Unidos, Reino Unido, Canadá y Australia suman 328 muestras de las 844 (41\%) lo que confirma que la lengua dominante sea el inglés. En la figura 4 se aprecian las cinco lenguas más usadas en la nominación de las bandas, cabe destacar que la segunda lengua es el latín. La organización es respecto de la posición que ocupa el elemento léxico del nombre. Así, observamos la predominancia del inglés por cualquiera de las otras lenguas, siendo la única que se encuentra hasta la quinta posición. Le sigue el latín que aparece hasta la tercera posición de elementos en el nombre, la siguiente es el griego, que sólo aparece en nombres simples, le siguen el español hasta la segunda posición y el francés, el cual presenta una ocurrencia en tercera posición (en el nombre de la banda holandesa Agua de Annique).

Fig. 4. Las lenguas de nominación (elaboración propia).

\begin{tabular}{|l|c|c|c|c|c|}
\hline Lengua & 1a Pos & 2a pos & 3a pos & 4a pos & 5a pos \\
\hline Alemán & 6 & 1 & & & \\
\hline Árabe & 2 & 2 & & & \\
\hline Camboyano & 1 & & & & \\
\hline Danés & 2 & & & & \\
\hline Desconocido & 6 & 5 & 1 & & \\
\hline Español & 16 & 6 & & & \\
\hline Finés & 2 & & & & \\
\hline Francés & 7 & 2 & 1 & & \\
\hline Gaélico & 2 & 1 & & & \\
\hline Griego & 27 & & & & \\
\hline Hebreo & 3 & 1 & & & \\
\hline Hindi & 1 & 1 & & & \\
\hline Holandés & 1 & 1 & 1 & & \\
\hline Inglés & 701 & 284 & 123 & 25 & 3 \\
\hline Italiano & 3 & 1 & 1 & & \\
\hline
\end{tabular}




\begin{tabular}{|l|c|c|c|c|c|} 
Khmer & & 1 & & & \\
\hline Latín & 54 & 13 & 2 & & \\
\hline Letón & 1 & & & & \\
\hline Lituano & 1 & & & & \\
\hline Noruego & 2 & & & & \\
\hline Portugués & 2 & 1 & & & \\
\hline Rumano & 1 & & & & \\
\hline Sueco & 2 & 1 & & & \\
\hline Tagalo & & 1 & & & \\
\hline
\end{tabular}

Es interesante observar la presencia del griego y el latín. Una de las razones que suponemos es su carácter místico y erudito, que conlleva un aura de misterio ${ }^{14}$. Lo que también es importante resaltar es la connotación negativa de casi todos los nombres. Como ejemplos tenemos las bandas: Absurdus, Acrimonious, Aeternam, Aeternus, AEtherial, Arcana Coelestia, Arcturus, Abortus, Absentia, Animus, Aeon Arcanum, Aeternitas, Amore Ad Lunam, Aurora Borealis, Adversus, todas ellas o con un sentido místico o negativo. El griego se presenta más como en griego clásico en nombres como Adamas, Archaios, Awrizis, Acheron, Acherontas, Andras, Apollyon, Aristeia, Athos, Acrostichon, Archontes o la combinación griego y latín Arkhon Infaustus. Las lenguas romance también resultan lenguas "exóticas" para no hispanohablantes y se observa en la

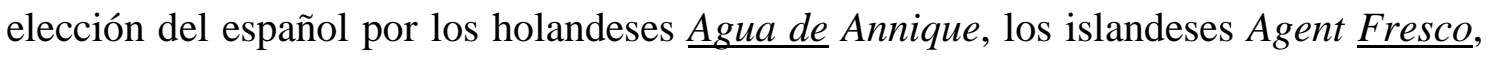
los americanos Altura o los fineses Antimateria; del francés por los noruegos Aura Noir, los brasileños Avec Tristesse, los estadounidenses Apathetiq y los austriacos Anomalie; solo Amesoeurs son franceses.

Las lenguas minoritarias presentan referencias interesantes como en árabe o en sueco por los estadounidenses Ana Kefr ${ }^{15}$ y $A s k a^{16}$, en danés o letón por los noruegos Arvinger $^{17}$ y Asaru $^{18}$ o en hindi por los nepaleses Antim Grahan ${ }^{19}$.

La clasificación en simples y compuestos se realiza conforme al número de elementos léxicos que contiene cada nombre. Sólo hay dos tipos: simples y compuestos,

\footnotetext{
${ }^{14}$ Este fenómeno se observa en varios contextos, como en la serie de hechizos de la saga Harry Potter de J. K. Rowling. El nombre de la banda Ava Inferi (Portugal), incluso, está basado en Los inferi (inferius), los cadáveres de humanos que han sido poseídos mediante magia oscura para hacer con ellos lo que se le antoje a un mago tenebroso. Consultado en https://harrypotter.fandom.com/es/wiki/Lista_de_hechizos

15 Ana Kefr - 'soy infiel'

${ }^{16}$ Cenizas

${ }^{17}$ Herederos

${ }^{18}$ Lágrimas

${ }^{19}$ Último eclipse
} 
entendiendo que los primeros son aquellos que sólo se componen de un elemento léxico y los segundos, aquellos que se componen de dos o más. Así, se obtiene 520 simples y 324 compuestos. Los nombres compuestos se dividen en cada uno de sus componentes, encontrando que el máximo es de cinco elementos, por lo cual se denominan Morf1... Morf5 a cada columna. En ellas se encuentran las categorías gramaticales (sustantivo, verbo, adjetivo, adverbio; preposición, artículo, pronombre, conjunción e interjección) a las que pertenecen (REVILLA DE COS, 2000) o a algunos procesos de formación de palabras entre los que hallamos abreviatura, acrónimo, compuesto léxico y creación léxica (YULE, 2004). A estos se suman los que fue difícil hallar información al respecto denominándolos "desconocido" pues es difícil determinar a qué categoría pertenecen. Para los fines de este trabajo, se decide separar una categoría denominada "nombre propio", la cual surge a partir de la revisión de cada una de las muestras.

Otra columna categoriza los compuestos de acuerdo con la estructura sintáctica (frase, oración, sintagmas nominales y preposicionales) (LOPE BLANCH, 1983: 13-14), "nombre completo" y "sin clase". Hay cinco columnas con la leyenda Lengua $1 . .$. Lengua5 para cada componente y la última es la que se refiere a la clasificación onomástica, con notas adicionales como el tipo de referencia (literaria, histórica, religiosa, etc) y datos enciclopédicos.

\section{Algunas características lingüísticas}

Respecto de la estructura lingüística, primero presentaremos la estructura morfológica y luego la sintáctica. En la primera veremos la aparición de categorías gramaticales y procesos de formación de palabras y en la segunda, las estructuras sintácticas observadas.

En primer lugar, vemos en la figura 5, al sustantivo, la categoría de mayor predominancia y que, en su mayoría, funcionan como elementos constitutivos de los nombres compuestos. Es la única categoría que ocupa las cinco posiciones en tres ocurrencias (Arsonists Get All the Girls, At the Throne of Judgment y And There Will Be $\underline{B l o o d})$. A continuación, tenemos al adjetivo el cual también aparece en la cuarta posición

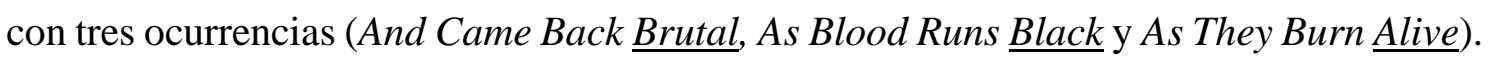
El compuesto léxico, entendido como la "concatenación de dos palabras separadas para producir una forma única” (YULE, 2004: 77), es el que sigue en número de ocurrencias con 37 en primera posición y 1 en segunda posición. Del segundo, el ejemplo es la banda 
estadunidense Alabama Thunderpussy y algunos ejemplos del primero son Abidetherein $^{20}$, Afterblood, Afterlight, Alexisonfire, Aliengates, Amongruins, Angelscourge, Annihilationmancer, Annisokay, Armsbendback, Aschenglas, Audiopain, y Axewound, etc.

Figura 5. Las categorías léxicas (elaboración propia)

\begin{tabular}{|l|c|c|c|c|c|}
\hline Elemento léxico & \multicolumn{1}{|c}{ la Pos } & \multicolumn{3}{l}{ 2a pos $3 \mathrm{a}$ pos $4 \mathrm{a}$ pos } \\
\hline Abreviatura & 7 & 2 & & & \\
\hline Acrónimo & 4 & & & & \\
\hline Adjetivo & 168 & 32 & 5 & 3 & \\
\hline Adverbio & 34 & 5 & 1 & & \\
\hline Artículo & 27 & 13 & 7 & 1 & \\
\hline compuesto léxico & 37 & 1 & & & \\
\hline Conjunción & 6 & 2 & & & \\
\hline creación léxica & 26 & & & & \\
\hline Desconocido & 50 & 6 & 1 & & \\
\hline Interjección & 2 & & & & \\
\hline nombre propio & 136 & 18 & 8 & & \\
\hline Numeral & & 3 & & & \\
\hline Preposición & 13 & 44 & 6 & 2 & \\
\hline Pronombre & 2 & 19 & 3 & & \\
\hline Sustantivo & 299 & 169 & 71 & 12 & 3 \\
\hline Verbo & 33 & 9 & 27 & 7 & \\
\hline
\end{tabular}

El nombre propio es una categoría constante, pero esta se dejará para la sección correspondiente, por lo tanto, continuaremos con los adverbios que aparecen hasta la tercera posición en el caso del nombre-frase de los canadienses As Bold As Lions que, desde el punto de vista fraseológico equivale a la alteración del símil "to be as bold as brass $^{21}$ ". Los verbos aparecen 33 veces en primera posición y representan por lo general una oración o una frase, pero también ocurren en segunda, tercera y hasta en cuarta posición en los nombres And There Will $\underline{B e}$ Blood, All that I Bleed, The Air I Breathe, At Dawn They Sleep, All Pigs Must Die. Los verbos que aparecen de manera individual se destacan por la connotación negativa de los elementos seleccionados como Abacinate (cegar a alguien con metal candente), Abandon, Agonize, Asunder.

\footnotetext{
${ }^{20}$ Frase del Corán: "The people of Hell will abide therein forever" [al-Jinn 72:23] "Verily, the Mujrimoon (criminals, sinners, disbelievers) will be in the torment of Hell to abide therein forever (Merriam-Webster's).

${ }^{21}$ Gran Diccionario Oxford. Tercera edición. Versión en CD-ROM (2003).
} 
Otros elementos léxicos como las preposiciones también aparecen en varias posiciones en la frase. Debido a los verbos frasales del inglés incluídos en los nombres como And Hell Followed with, o At the Throne of Judgment, las preposiciones aparecen como el cuarto elemento del nombre. Cabe destacar que hay 50 muestras denominadas "desconocido", debido a que no fue posible encontrar un significado o una referencia. Las creaciones léxicas tienen explicación en las páginas de las bandas o en la enciclopedia de Metal y por lo general se debe a que se cambia un elemento mínimo de la palabra para generar una nueva. Hay ciertos elementos también que nos ayuda a identificarlos como el caso de Asgaroth (sólo una variante de Esgaroth) o Andragonia (en el que identifican dos elementos como Andros o Dragonia). A diferencia de los anteriores ejemplos, en "desconocido" no es posible hallar referencias ni información y en los diccionarios o la red no hay información adicional, por lo que desconocemos si se trata de una modificación a una palabra o es una palabra que han creado los miembros de la banda.

En relación con la sintaxis, se han considerado todas las estructuras que se forman a partir de dos elementos. Lope Blanch $(1983,1990)$ propone una tipología de estructuras entre las que encontramos la oración, la pro-oración, la frase, el periodo y la cláusula. Pero hemos tomado una tipología fraseológica y algunas categorías las hemos decidido personalmente como el nombre completo, aunque es una construcción de hasta tres elementos, este solo representa, semánticamente a uno. Siguiendo la teoría de Charles Bally (en TIMOFEEVA, 2008: 95; en CARNEADO y TRISTÁ, 1989: 7), consideramos unidades no descomponibles (unités indécomposables) como las locuciones ${ }^{22} \mathrm{y}$ agrupaciones cambiantes (groupements passagers), sintagmas que muestran grados de descomposicionalidad, entre los que consideramos la frase, la oración y sintagmas nominales y preposicionales, además de una categoría "sin clase" al no ser posible la clasificación. Clasificamos como "nombre completo" a las agrupaciones complejas de dos o tres elementos que contienen un nombre de persona y uno o dos apellidos, que se explican en la siguiente sección.

Figura 6. Las estructuras sintácticas presentes en el corpus (elaboración propia)

\begin{tabular}{|l|r|}
\hline Frase & 34 \\
\hline Locución & 4 \\
\hline $\begin{array}{l}\text { Nombre } \\
\text { completo }\end{array}$ & 16 \\
\hline
\end{tabular}

22 "Combinación inicial con mayúscula fija de varios vocablos que funciona como una determinada clase de palabras” (DRAE, 2020). 


\begin{tabular}{|l|r|}
\hline Oración & 56 \\
\hline Sin clase & 4 \\
\hline Sint. Nominal & 206 \\
\hline Sint. Prep. & 4 \\
\hline
\end{tabular}

\section{De los aspectos onomásticos en los nombres de las bandas}

En esta sección presentamos el análisis de los 172 crematónimos derivados de un nombre propio. De nuevo retomamos el concepto de relación intercategorial (LÓPEZ FRANCO, 2020: 232) pues el nombre propio pasa a nombrar un objeto. Para el análisis, presentaremos las categorías más frecuentes y dejaremos los menos frecuentes al final siguiendo la representación gráfica de la figura 7.

Figura 7. Las categorías onomásticas

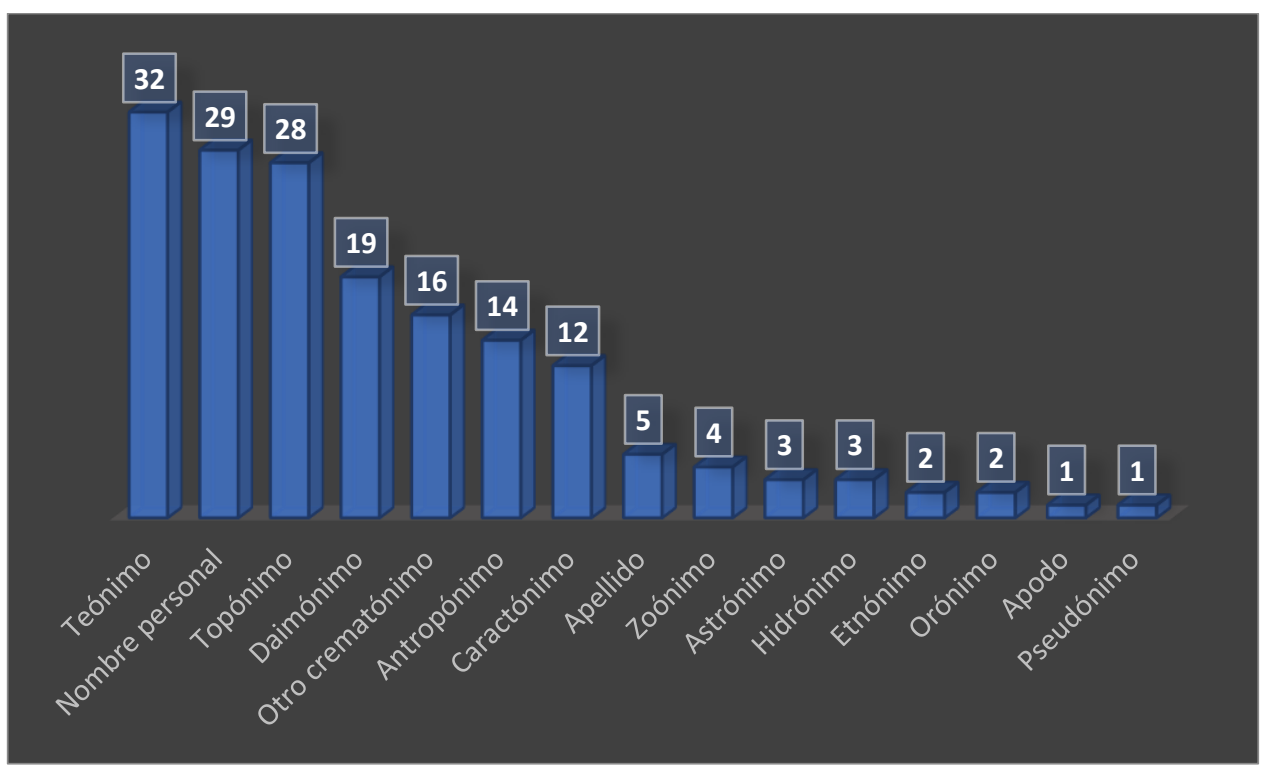

\section{Teónimo-Daimónimo}

La categoría más frecuente es la de los teónimos, que se han separado de los daimónimos o nombres de un demonio o contraparte de un ser de carácter sagrado, al cual se le conoce como daimonia (AYNARD, 1999: 204) y de este vocablo deriva daimónimo, concepto difícilmente localizable, pues solo es mencionado por Montaner y Lara (2014: 92) y que aceptamos y proponemos en este trabajo. En nuestro corpus encontramos diversos orígenes de los nombres, seguramente con el afán de exotismo, pero también de individualizar en la nominación alejándose de lo inmediato o, por lo contrario, rescatando lo local, tal vez como signo de identidad (el caso de los fineses Aarni, o los alemanes Aaskereia quienes usan un teónimos de raíz nórdica). La tradición griega es la más 
representada y las ocurrencias son Adastreia, Aegaeon, Age of Artemis, Age of Nemesis, Aion, Antaeus, Apollyon (2), Ares Kingdom, Atena, Athena, Ashes of Ares y Athos. A estos les sigue la tradición egipcia Amenra, Amon, Amset, Anubi, Anubis Gate y Apophis. Otros orígenes son el gaélico y celta con los Aes Dana y Asrai, respectivamente o el nombre de los franceses Ases (plural de dios en islandés). Otros orígenes más orientales y menos comunes son Ahoora (hindú), Alkonost (eslavo), Anael (judaico), Angizia (Umbro), Astarte (asirio), Atargatis (arameo), Aurora (latino) y Azrael (bíblico).

Hallamos 19 nombres de demonios; cuatro de ellos derivan de la tradición bíblica

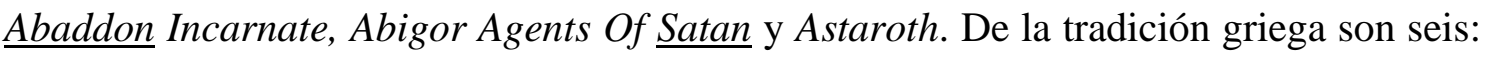
Abraxas (2) Agathodaimon, Akheron, Akhlys y Alastor Sanguinary Embryo y de otras culturas encontramos a Adramelech (demonio de la cultura asiria), Aesma Daeva (de origen avéstico), Ahriman (del zoroastrismo), Ajattara (nórdica), Anunnaki (daimónimo sumerio), Alukah y Asmodée (hebrea) y uno de tradición Inuit: Amarok.

\section{Antropónimos - nombres - apellidos}

Los nombres agrupados en la denominación "antropónimo complejo" son en realidad la combinación Nombre de persona + apellidos. Por lo general se refieren a un miembro o líder de la banda y los más comunes son Nombre + apellido: Ace Augustine, Ace Frehley, Alice Cooper, Amadeus Awad, Andi Deris, Andre Andersen, Andre Matos, Andrea Gail y Arno Rush, además de Abigail Williams, que no se refiere a un líder de la banda, sino a un personaje histórico; se trata de los primeros acusadores de los Juicios de Salem que llevó al arresto y prisión de más de 150 personas acusadas de brujería, al mismo tiempo es un caractónimo en la obra de teatro The Crucible de Arthur C. Miller (STEVENSON, 2008). Existen cuatro ocurrencias con tres componentes: Andrew W.K. ${ }^{23}$ (con iniciales), Ardulph Ardebahr Wald (parece ser invención nominal), Arjen Anthony Lucassen y Axel Rudi Pell.

Los nombres individuales son Adaliah, Adelaide, Adema, Adestria, Agamendon, Agathocles, Alaya, Alister, Amaranthe, Amos, Angantyr, Angus Argus, Archagathus, Arsames, Artemesia, Arthemesia, Asterion, Attila, Abdullah, Abigail, Abramelin, Aina y Andras. La mayoría de ellos son personajes históricos o con referencia histórica, a partir

\footnotetext{
${ }^{23}$ De nombre Andrew (Fetterly) Wilkes-Krier (https://es.wikipedia.org/wiki/Andrew_W.K.)
} 
de referencias en diccionarios o información en la red. El resto aparece en compuestos como Agua de Annique, Alexisonfire, Adamus Exul, Alice in Chains, Alice in Darkland.

También hay casos que solo llevan apellido o apellidos, son cinco: Adgar (apellido de Willame, o Adgar William o Edgar, son los nombres antiguos que se dan a un escritor medieval inglés de la segunda mitad del siglo XII que utilizó el idioma anglonormando para redactar sus obras ${ }^{24}$ ), Alighieri, de clara alusión literaria, Assel y los compuestos Arch / Matheos ${ }^{25}$ y Allen / Lande ${ }^{26}$.

\section{Topónimos}

En esta categoría encontramos referencias interesantes pues en su mayoría son referentes o literarios o religiosos y de lenguas diversas. Los polacos $A l$ sirat usan el topónimo coránico para nombrar a su banda, se refiere al delgado puente por donde pasa el alma para entrar al paraíso. Empecemos por los topónimos con alusión geográfica como Alabama Thunderpussy, Almora, Aorlhac ${ }^{27}$, Arkona $^{28}$ (2), Astrakhan, Attika, Azusa, ${ }^{29}$ Asking Alexandria. Algunos nombres aluden a referencias mitológicas o religiosas de diverso origen como Amarna Reign (egipcia), Angara Damana (zoroastrismo), Angkor Wat (jemer), Arallu ${ }^{30}$ (mesopotámica), Avichi ${ }^{31}$ (budista) y Asgaard, Asgarth, Aasgard (topónimo de la mitología nórdica). Albion / Tharotia y Albion (el nombre más antiguo de la Gran Bretaña), Arkham Witch (de la obra de H. P. Lovecraft), Avalon (de la mitología celta), Azarath ${ }^{32}$, Arthedain (reino de los Edain, cuya capital es Fornost (FOSTER, 2002: 39-40) son topónimos de inspiración literaria. Quedan

\footnotetext{
${ }^{24}$ https://es.wikipedia.org/wiki/Adgar

25 Reunión entre los fundadores: John Arch y Jim Matheos miembros de Fates Warning. https://en.wikipedia.org/wiki/Arch/Matheos (Traducción propia)

${ }^{26}$ Allen/Lande es un proyecto de heavy metal melódico formado en 2005 por el guitarrista Magnus Karlsson (Primal Fear, Starbreaker) con dos conocidos vocalistas: el cantante de Masterplan Jørn Lande y el de Symphony X Russell Allen. Las canciones, todas escritas por Magnus Karlsson, muestran rasgos de hard rock y el metal progresivo (https://es.wikipedia.org/wiki/Allen/Lande) (Traducción propia)

${ }^{27}$ Pronunciación [ur' Kak]). Se refiere a Aurillac, el lugar de origen de la banda. Se inspira en el nombre del lugar en lengua occitana, Orlhac. https://www.metal-archives.com/bands/Aorlhac/3540264432 (Traducción propia)

${ }^{28}$ Arkona fue la última ciudad-fortaleza pagana eslava en ser destruida en 1168 por la cruzada lidereada por el Obispo Absalon y el rey Valdemar el Grande de Dinamarca. https://www.metalarchives.com/bands/\%D0\%90\%D1\%80\%D0\%BA\%D0\%BE\%D0\%BD\%D0\%B0/26171 (Traducción propia)

${ }_{29}$ Azusa, fundada en 1898, es una ciudad ubicada en el condado de Los Ángeles en el estado de California. https://es.wikipedia.org/wiki/Azusa_(California)

${ }^{30}$ En la mitología Mesopotámica "Arallu" es el reino subterráneo regido por la diosa Ereshkigal y el dios Nergal donde se juzga a los muertos. https://www.metal-archives.com/bands/Arallu/3059 (Traducción propia)

${ }^{31}$ En el budismo, Avīci (en sánscrito y pali: 'sin ondas', 'sin olas') es el nivel más bajo del Reino Naraka o

"infierno", en el cual un ser que ha cometido graves fechorías puede renacer. https://es.wikipedia.org/wiki/Av\%C4\%ABci

32 Azarath, es una dimensión ficticia creada por DC Comics para la serie Teen Titans, y a la que también se hace referencia en su serie animada. Azarath es el lugar de nacimiento de Raven, uno de los miembros de los Teen Titans. https://es.wikipedia.org/wiki/Azarath (Traducción propia)
} 
Avantasia, según la banda, es la mezcla de Avalon + Fantasia y Azeroth (2), topónimo ficticio en el mapamundi de World of Warcraft $(1994)^{33}$.

\section{Crematónimos derivados de otro crematónimo}

Otra categoría que debe enfatizarse son los derivados de otro crematónimo, es decir, de aquellos que ya representaban un nombre propio en esta categoría y que se reciclan para ser usados para nombrar a las bandas ${ }^{34}$. De esta categoría se hallaron 15 ocurrencias. En primera instancia aparece $A b a c a b b^{35}$, Aeveron (topónimo ficticio mencionado en la canción Fields od Aeveron ${ }^{36}$ ). Cuatro son canciones de otras bandas Abyssic Hate (Darkthrone), After Forever (Black Sabbath), All That I Bleed (Savatage), Alma mater (Moonspell) y tres son nombres de álbumes Astral sleep (Tiamat), Alternative 4 (Anathema) y Atoma (Dark Tranquility). Con referencias culturales tenemos a Ark of the Covenant (de origen bíblico), Andralls ${ }^{37}$ Arion $^{38}$ (mitología) y del cine y la literatura tenemos a Abydos (topónimo derivado de Abidos o Abydos ${ }^{39}$, Ador, Alustrium ${ }^{40}$, And there will be blood ("Pozos de ambición en español", Película de Paul Thomas Anderson, $2007^{41}$ ) y Asangreyfuego (“A sangre y fuego", trilogía novelística escrita por Henryk Sienkiewicz, $1884^{42}$ ).

\section{Caractónimos}

El caractónimo se refiere, según ICOS al nombre de personajes de obras literarias, principalmente en esta categoría encontramos trece ocurrencias, en su mayoría personajes

\footnotetext{
${ }^{33}$ Azeroth - www.wowpediawow-es.gamepedia.com/Azeroth

${ }^{34}$ Este fenómeno se presenta no solo en el caso de las bandas, solo por mencionar algún ejemplo, tenemos Soul Kitchen, película cuyo nombre proviene de la canción homónima compuesta por Jim Morrison.

${ }^{35}$ A-B-A-C-A-B-B es el Código de entrada que habilita los efectos sangrienteos/gore para el videojuego original Mortal Kombat (de la versión Sega Genesis). Es también el Código se nombró "Abacabb" debido al álbum Abacab de la banda de rock progresivo Genesis. https://www.metal-archives.com/bands/ABACABB/6589 (Traducción propia)

${ }^{36} \mathrm{https}: / /$ www.musica.com/letras.asp?letra $=1826836$

${ }^{37}$ Andralls se basa en el nombre de Andraus, un edificio que colapsó en un incendio en 1972, matando a 16 personas e hiriendo a otras 330 en São Paulo, ... https://www.metal-archives.com/bands/Andralls/5125 (Traducción propia)

${ }^{38}$ En la mitología griega Arión era un fabuloso caballo de crines negras que podía correr tan rápido que podía ir sobre el agua y tenía estirpe divina. Poseidón, dios del mar y de los caballos, intentó en una ocasión seducir a su hermana Deméter, sin conseguirlo. https://es.wikipedia.org/wiki/Ari\%C3\%B3n_(mitolog\%C3\%ADa)

${ }^{39}$ Pueden ser Abidos, en el Antiguo Egipto, Abido o Abidos, antigua colonia griega del Helesponto, Abidos (Pirineos Atlánticos), comuna de Francia o Abidos o Abydos, planeta ficticio de la serie Stargate SG-1 (probablemente sea esta última la referencia) https://es.wikipedia.org/wiki/Abidos_(desambiguaci\%C3\%B3n)

40 quizá a la obra de Jovius, P. Allustrium Virorum Elogia. Único referente hallado en la red. https://books.google.com.mx/books?id=ges_AAAAIAAJ\&pg=PA193\&lpg=PA193\&dq=Allustrium\&source=bl\&ots =tICWZ7SjzM\&sig=ACfU3U0M4dOFsw-Rv680cI-

AzjSuG6Ufiw\&hl=es\&sa=X\&ved=2ahUKEwj9xbScw5zsAhXVaM0KHWdXCIEQ6AEwBHoECAgQAg\#v=onepa ge $\& \mathrm{q}=$ Allustrium $\& \mathrm{f}=$ false

${ }^{41}$ https://es.wikipedia.org/wiki/There Will Be Blood\#: :text=There\%20Will\%20Be\%20Blood\%20es,en\%201927\% 20por\%20Upton\%20Sinclair

42 https://es.wikipedia.org/wiki/A_sangre_y_fuego_(trilog\%C3\%ADa_polaca)
} 
literarios: Ahab (alusión al Capitán Ishmael Ahab de la novela Moby Dick de Herman Melville), Akela (Rudyard Kipling, The Jungle Book), Alhana ${ }^{43}$, Atreyu (Michal Ende, La historia interminable), Dorath ${ }^{44}$ y cuatro personajes del universo de J. R. R. Tolkien Arathorn (Padre de Aragorn, rey de Gondor), Arven ${ }^{45}$ y Arwen (Elfa hija de Elrond, esposa de Aragorn) y Azaghal (un rey enano) ${ }^{46}$; tres de videojuegos: Altaria (Pókemon), Atem (Nameday) y Annthennath (El Rompecabezas del Milenio), así como Ashtar ${ }^{47}$, que es de la cultura popular.

\section{Otras categorías}

Finalmente, presentamos las categorías con menor número de ocurrencias. Tenemos dos categorías que son un tanto controversiales; uno es el de los zoónimos (nombres de animales) y el de los etnónimos ya que en español los gentilicios no se representan con la mayúscula como en inglés, pero, dado que la mayoría de los nombres están en inglés se han conservado. Los zoónimos son cuatro: Abyssaria, Acrassicauda, Archons y the ATLAS MOTH, de los cuales, sólo el último contiene un nombre propio (el teónimo Atlas). A estos siguen los casos que contienen astrónimos o nombres de cuerpos celestes en las bandas Altar of Betelgeuze, Andromeda y Arcturus. Existen tres hidrónimos o nombres de masas de agua y las bandas son Absu (un océano subterráneo en la mitología sumeria), Acheron y Acherontas (el río del Hades). Encontramos a Abkanis $^{48}$ y Amaseffer $^{49}$ como ejemplos de etnónimos; los dos orónimos son de la mitología de Tolkien, estos son Amon Amarth (Monte del destino) y Amon Din (FOSTER, 2002: 13). Por último, hay un apodo, el de Abbath (apodo de Olve Eikemo o Abbath Doom Occulta fundador de la banda Immortal) y un pseudónimo, que decidimos

\footnotetext{
${ }^{43}$ Alhana Starbreeze es un personaje del universo ficticio de Dragonlance. Es una elfa silvanesti del continente de Ansalon. https://es.wikipedia.org/wiki/Alhana_Starbreeze (Traducción propia).

${ }^{44}$ Adorath era un mercenario espadachín, líder de una banda llamada "Compañía de Dorath", que vivía en los campos rondando la Colina Cantrevs de Prydain (https://prydain.fandom.com/wiki/Dorath); las Crónicas de Prydain es una saga de cinco novelas de alta fantasía escrita por Lloyd Alexander. https://es.wikipedia.org/wiki/Las_Cr\%C3\%B3nicas_de_Prydain (Traducción propia)

45 Pronunciación alemana

${ }^{46}$ Robert Foster, 2002

${ }^{47}$ Ashtar es el nombre dado a un ser extraterrestre o a un grupo de seres con los cuales un número de personas afirman haberse canalizado. George Van Tassel, un ciudadano norteamericano, afirma ser el primero en haber recibido un mensaje en el año 1952, proveniente de Ashtar. https://es.wikipedia.org/wiki/Ashtar (extraterrestre)

$48 \mathrm{La}$ tribu Abkani Tribe era una civilización nativo Americana que vivía en La Isla de las Sombras https://aloneinthedark.fandom.com/wiki/Abkani_tribe), etnónimo ficticio que aparece en Alone in the Dark: The New Nightmare, originalmente conocido como Alone in the Dark 4, es la cuarta entrega de la saga de survival horror Alone in the Dark creado por Darkworks y publicada por Infogrames. https://aloneinthedark.fandom.com/es/wiki/Alone in the Dark: The New Nightmare

49 Amaseffer (עַם הַספספר) es el nombre Hebreo que se refiere al escogido por los Israelitas en la biblia y signifca "Pueblo del Santo Libro" https://en.wikipedia.org/wiki/Amaseffer (Traducción propia).
} 
considerar así el nombre de la banda argentina Almafuerte pues quizá sea un homenaje al escritor argentino Pedro Bonifacio Palacios.

Esta variedad de referencias vertidas en un nombre comercial de una agrupación es un entramado de conexiones sociales, lingüísticas y culturales y que tiene repercusiones lingüísticas (se convierten en nuevos referentes), sociales (pueden tener influencia en el proceso de reconversión de referentes al existir la posibilidad de que un nombre de banda lo sea de una persona, mascota, etc.) y culturales (ensanchan los referentes de la subcultura metalera).

\section{Conclusiones}

El crematónimo es un concepto que necesita estudiarse desde las más diversas ópticas puesto que individualiza objetos que, de otra forma, estarían perdidos en la historia o en el olvido. Para la onomástica es de sumo interés conocer no solo el nombre, sino las motivaciones y las referencias culturales, literarias, histórica y, en su caso, personales para entender el proceso cognitivo de nombrar. En este trabajo se ha presentado el caso de los nombres de bandas de Heavy Metal desde un punto de vista exploratorio bajo el análisis lingüístico-onomástico. El género es considerado una subcultura con rituales, modas, ideologías y discursos propios y a estos se ciñen los nombres de las bandas. Con este análisis no solo encontramos que la influencia nominal proviene de fuentes mitológicas, religiosas, literarias, históricas y lingüísticas. En el afán de dar el sentido de unicidad, que también deberá estar asociado a las reglas y rituales del $\mathrm{HM}$, se eligen nombres con connotación negativa y algunos en lenguas que dan el sentido de exotismo y misterio.

En el aspecto onomástico se han encontrado influencias diversas que van de antropónimos y apellidos hasta teónimos y daimónimos (concepto que se propone para distinguir la naturaleza divina y no divina de la referencias teológicas) pasando por apodos, toopónimos, caractónimos, creamatónimos y categorías menores como los zoónimos, etnónimos y astrónimos. La influencias de estos referentes entre los usuarios de la lengua y los seguidores del género y la influencia de los representantes de este género en la sociedad da origen a un continuo flujo de relaciones intercategoriales: los nombres de personas nombran entes comerciales y es posible que estos nombres terminen en algún momento (si no es que ya sea realidad) nombrando a personas en un proceso a 
la inversa. Dejamos abierta la invitación a abrir los oídos a nuevos conceptos y a nuevos horizontes onomásticos.

Recebido em 09/10/2020

Aceito em 04/11/2020

Publicado em em 17/12/2020

\section{Referencias}

(2003). Gran Diccionario Oxford. Tercera edición. Versión en CD-ROM. Oxford: Oxfod University Press.

(2020) Metal Archives. Encyclopaedia Metallum. Disponible en https://www.metalarchives.com. Consultado el 7 de noviembre de 2019

(2020). Behind the Name, the etymology and history of first names. Disponible en www.behindthename.com

Aldrin, E. (2011) Namnval som social handling. Val av förnamn och samtal om förnamn bland föräldrar i Göteborg 2007-2009. Uppsala: Institutionen för nordiska språk.

Angeler, D. G. (2016) "Heavy metal music meets complexity and sustainability science”. In SpringerPlus 5:1637 DOI 10.1186/s40064-016-3288-9

Aymard, R. (1999) « Hagiotoponymie de la Grèce » en Nouvelle revue d'onomastique, $\mathrm{n}^{\circ}$ 33-34, pp. 191-234. DOI: https://doi.org/10.3406/onoma.1999.1342 Disponible en $\quad$ https://www.persee.fr/doc/onoma_0755-7752_1999_num_33_1_1342. Consultado el 2 de octubre de 2020.

Boerrigter, R. \& Nijboer, H. (eds.) (2012) Names as Language and capital. Proceedings. Names in the Economy III, Amsterdam, 11-13 June 2009. Amsterdam: Meertens Instituut.

Caffarelli, E. (2011). "Nomi Commerciali” en Enciclopedia dell'Italiano. Disponible en: http://www.treccani.it/enciclopedia/nomi-commerciali_(Enciclopediadell'Italiano)/

Carneado Moré, Z. y Tristá, A. M. (1989). Estudios de Fraseología. La Habana: Academia de Ciencias de Cuba-Instituto de Literatura y Lingüística. 
Onomástica Desde América Latina, n.3, v.2, janeiro - junho, 2021, p. 59 - 81 ISSN 2675-2719 https://doi.org/10.48075/odal.v0i0.26062

Dark lyrics (2019) Dark Lyrics. Bands A. Disponible en http://www.darklyrics.com/a.html Consultado el 25 de octubre de 2019

Foster, R. (2002) Guía Completa de la Tierra Media. 2 tomos (A-G, H-Z). Madrid: Planeta de Agostini-Minotauro. Colección Biblioteca Tolkien

González-del Río, J., Ampuero Canellas, O., Jordá Albiñana, B. y Magal Royo, T. (2011) "El nombre de marca: interrelación de factores lingüísticos y corporativos". En Revista de Lingüística y Lenguas Aplicadas, vol. 6, pp. 181-193. DOI: http://dx.doi.org/10.4995/rlyla.2011.902

Hadžajlić, H. (2018) “Heavy Metal and Globalization” en AM Journal, No. 17, 129-137. DOI: http://dx.doi.org/10.25038/am.v0i17.276

International Council of Onomastic Sciences (2020) Onomastic terminology. Disponible en https://icosweb.net/drupal/terminology

Jiménez Segura, S. (2020) "La construcción de la identidad de género a partir de la selección del nombre de pila” en Onomástica desde América Latina, n. 1, v. 1, 2020, pp. 168-191.

Kallio, M. (2016) "Finnish Female Name Pattern with the Suffix -iina" en Carole Hough and Daria Izdebska (eds). Names and Their Environment. Proceedings of the 25th International Congress of Onomastic Sciences, Glasgow, 25-29 August 2014. Vol. 3, pp. 144-153

Lope Blanch, J. M. (1983). Análisis Gramatical del Discurso. México: Universidad Nacional Autónoma de México.

Lope Blanch, J. M. (1990) "El análisis gramatical de la cláusula" en Violeta Demonte y Beatriz Garza Cuarón (Eds.) Estudios de lingüística de España y México. México: El Colegio de México-IIF-UNAM. pp. 259-272. DOI 10.2307/j.ctv43vs5t.14. Disponible en http://www.jstor.com/stable/j.ctv43vs5t.14

López Franco, Y. G. (2020). "Las relaciones intercategoriales e intracategoriales en antroponimia. El caso de los nombres de pila en francés de Francia y en español de México" en Onomástica Desde América Latina, n.1, v.1, 2020, p. 214-239. 
Mayer, A. and Jeffrey M. Timberlake, J. M. (2014) ““"The Fist in the Face of God”: Heavy Metal Music and Decentralized Cultural Diffusion” en Sociological Perspectives, Vol. 57(1) 27-51. DOI: 10.1177/0731121413516607

Montaner, A. y Lara, E. (2004) "Magia, hechicería, brujería: deslinde de conceptos" en Eva Lara y Alberto Montaner (coords.) Señales, Portentos y Demonios. La magia en la literatura y la cultura españolas del renacimiento. Salamanca: LA SEMYR, pp. 33-184.

Phillips, W. and Cogan, B. (2009). Encyclopedia of Heavy Metal Music. Westport, Connecticut: Greenwood Press

Poutiainena, A. and Esa Lilja, E. (2012) "Heavy Metal and Music Education” en Procedia Social and Behavioral Sciences, 45, 517-526 DOI: 10.1016/j.sbspro.2012.06.589

Progarchives (2020) Progressive Rock Music Bands/Artists List Starting with letter [C]. Disponible en https://www.progarchives.com/bands-alpha.asp?letter=c

Real Academia Española (2020). Diccionario de la Real Academia Española. https://dle.rae.es/

Rengifo García, E. (2013). "El nombre comercial”. En Revista de la propiedad inmaterial, 17, pp. 187-203.

Revilla de Cos, S. (2001). Gramática española moderna. Un nuevo enfoque. Segunda edición. México: McGraw-Hill

Reyes Contreras, M. (2019) “Onomástica y branding. Dos formas de conocer la vitalidad de la lengua a través de la designación del negocio familiar". En Juan Carlos Escamilla y Felipe Bustos (comps.) Evidencias de investigación y docencia en el área de Lenguas. Ciudad de México: UNAM, FES-Zaragoza, p. 61-75

Stevenson Muñoz, R. (2008) “Abigail Williams as a femme fatale in The Crucible” en ES, 28, pp. 213-223.

Timofeeva, L. (2008) Acerca de los aspectos traductológicos de la fraseología española. Alicante: Universidad de Alicante. Tesis Doctoral

Tort i Donada, J. \& Montagut i Montagut, M. (eds.) (2014). “Introducción”. En Els noms en la vida quotidiana. Actes del XXIV Congrés Internacional d'ICOS sobre 
Onomástica Desde América Latina, n.3, v.2, janeiro - junho, 2021, p. 59 - 81 ISSN 2675-2719 https://doi.org/10.48075/odal.v0i0.26062

Ciències Onomàstiques. Barcelona: Generalitat de Catalunya-Departament de Cultura-Direcció General de Política Lingüística.

Yule, G. (2004). El Lenguaje. Cuarta edición. Madrid: Ediciones Akal. 